\title{
Robust Fault Detection for Vehicle Lateral Dynamics: A Zonotope-based Set-membership Approach
}

\author{
Sara Ifqir ${ }^{1}$, Vicenç Puig ${ }^{2}$, Naima Ait-Oufroukh ${ }^{1}$, Dalil Ichalal $^{1}$ and Saïd Mammar ${ }^{1}$
}

\begin{abstract}
In this work, a model-based fault detection layout for vehicle lateral dynamics system is presented. The major focus in this study is on the handling of model uncertainties and unknown inputs. In fact, the vehicle lateral model is affected by several parameter variations such as longitudinal velocity, cornering stiffnesses coefficients and unknown inputs like wind gust disturbances. Cornering stiffness parameters variation is considered to be unknown but bounded with known compact set. Their effect is addressed by generating intervals for the residuals based on the zonotope representation of all possible values. The developed fault detection procedure has been tested using real driving data acquired from a prototype vehicle.

Index Terms - Robust fault detection, interval models, zonotopes, set-membership, switched uncertain systems, LMIs, input-to-state stability, arbitrary switching.
\end{abstract}

\section{INTRODUCTION}

Detection of malfunctions and faults in vehicle lateral dynamics is one of the most important tasks allowing to improve the performance and reliability of active safety systems. An early warning indicating the occurrence of faults can help to take appropriate actions and avoid damage or even fatal accidents. Therefore, it is necessary to develop a Fault Detection (FD) procedure to be able to detect potential faults as early as possible. Then, the system can maintain stability and keep acceptable performances by means of this early warning including some fault tolerant mechanism.

Among numerous FD techniques, model-based design has attracted an increasing amount of attention over the last decades, and some approaches have been proposed (see, e.g. [3], [7], [10], [14], [15] and references therein). They perform fault detection by generating a residual signal based on the difference between the real and mathematical model outputs, which in the fault-free case should be zero, otherwise, a fault is detected and an alarm is generated. However, since there exist some mismatches between mathematical model and real plant due to parameter uncertainties, external disturbances and unknown inputs, the residual signal becomes nonzero, which generates false alarms, corrupting the results and making the FD system useless. Hence, robustness against these uncertainties and disturbances is an important and challenging problem in model-based FD design.

In the literature, two main distinct approaches have been addressed. The first robust approach, known as active, is

\footnotetext{
1 Sara Ifqir, Naima Ait-Oufroukh, Dalil Ichalal and Saïd Mammar are with IBISC Laboratory, Univ Evry, Paris-Saclay University, Evry, France. \{sara.ifgir, naima.aitoufroukh, dalil.ichalal, said.mammar\}euniv-evry.fr

2 V. Puig is with Automatic Control Department, Universitat Politècnica de Catalunya, Barcelona, Spain. vicenc.puig@upc.edu
}

based on generating residuals that are sensitive to faults, while being insensitive to uncertainties. This approach has been extensively developed using Unknown Input Observer (UIO) [14], parity-space and identification based methods [2]. Whereas, the second approach, known as passive, is based on enhancing residual robustness at the decisionmaking stage using an adaptive threshold [10]. In other words, the passive approach consists on propagating the uncertainties to the residuals limits. When the residual is outside these limits, the alarm is not due to uncertainties but to a fault occurrence.

In the Passive Fault Detection (PFD) technique, uncertain parameters are bounded and the resulting model known as interval model is used for design. In this case, the measured output is bounded by an interval, providing, therefore, an interval for the residual vector. Then, fault detection test consists in checking if the residual value is inside or not this interval after uncertainty propagation. In this paper, zonotopes are used to provide the interval enclosing the unknown true residual vector. The advantage of using zonotopes is that monotonicity property is no longer needed as in [8], [9], [11] allowing to consider the whole set of possible trajectories. The zonotope-based approaches in fault detection has already been investigated in the literature, see for instance [5], [7]. The main contribution of this paper is that it provides a robust PFD schema based on the set-membership approach using zonotopes to bound the parameter uncertainty affecting the vehicle lateral dynamics. For this purpose, it is important to use a detailed and accurate vehicle model. In this study and in order to fully make use of the range variations of all vehicle uncertain parameters, such as, vehicle longitudinal velocity, cornering stiffness coefficients, and to take into account external disturbances such as lateral wind gusts, a switched uncertain discrete-time system subject to unknown inputs is proposed. In this model, the longitudinal velocity is considered piecewise constant, different local sub-model are then derived and a switching strategy dependent on the measured velocity is implemented. Furthermore, an adaptive dynamic tire model that is physical and flexible enough to permit timevarying tire performances is used. Indeed, a readjustment variable assumed to be unknown but bounded is added to correct the value of the cornering stiffness coefficients in case that a change occur in road adhesion or vehicle loading condition. Using Switched Unknown Input Observer (SUIO) principle and Input-to-State Stability concept, the residual vector is made robust against unknown lateral wind gusts disturbances and aforementioned uncertainties. This work 
shows the successful application of PFD method in order to detect faults in vehicle lateral dynamics. It should be pointed out that the use of adaptive threshold in vehicle lateral diagnosis systems is an area that is still fairly undeveloped. Moreover, for the best of the author knowledge, the PFD method has not been intensively investigated for switched systems. This paper, presents perhaps the first attempt to deal with such systems.

The remainder of this paper is organized as follows: Section II presents the vehicle lateral model under consideration. The design of the PFD schema is proposed in Section III. Application to vehicle lateral dynamics with validation through real data is given in Section IV. The main conclusions and future works are drawn in Section V.

\section{VEHICLE LATERAL DYNAMiCS MODELING}

In the literature, there are several mathematical models describing the vehicle lateral dynamics. In the present work, the well known two degree of freedom bicycle model representing the lateral and yaw motions is used for implementing FD procedure. The vehicle mass is equal to $m$. The moment of inertia is noted $I_{z}$. The front and rear axles are located respectively at distances $l_{f}$ and $l_{r}$ from the center of gravity. The front and rear lateral tire forces $F_{y f}$ and $F_{y r}$ are functions of wheel slip angles $\alpha_{f}$ and $\alpha_{r}$, respectively. The force $F_{w}$ is due to the effect of the wind gusts that are acting at a distance $l_{w} \cdot v_{y}$ and $v_{x}$ are lateral and longitudinal velocity respectively, and $\delta_{f}$ stands for the front steering angle. The vehicle lateral dynamics is described by the following differential equations with lateral velocity $v_{y}$ and yaw rate $\dot{\psi}$ as states [12]:

$$
\left\{\begin{array}{l}
m \dot{v}_{y}=F_{y f}+F_{y r}-m v_{x} r+F_{w} \\
I_{z} \ddot{\psi}=l_{f} F_{y f}-l_{r} F_{y r}+l_{w} F_{w}
\end{array}\right.
$$

For normal driving situations, $F_{y f}$ and $F_{y r}$ are linear with respect to wheel slip angles

$$
F_{y f}=c_{f} \alpha_{f}, \quad F_{y r}=c_{r} \alpha_{r}
$$

where $c_{f}$ and $c_{r}$ represent the front and rear wheel cornering stiffness parameters respectively. The front and rear wheel slip angles are defined as

$$
\alpha_{f}=\delta_{f}-\frac{v_{y}}{v_{x}}-\frac{l_{f}}{v_{x}} \dot{\psi}, \quad \alpha_{r}=-\frac{v_{y}}{v_{x}}+\frac{l_{r}}{v_{x}} \dot{\psi}
$$

Substituting (2) and (3) into (1), equations (1) are expressed in the following state-space form

$$
\begin{aligned}
& \dot{x}(t)=A x(t)+B u(t)+E d(t) \\
& y(t)=C x(t)
\end{aligned}
$$

where $x(t)=\left[\begin{array}{ll}v_{y} & \dot{\psi}\end{array}\right]^{T}, u(t)=\delta_{f}, d(t)=F_{w}, y(t)=\dot{\psi}$

$$
A=\left[\begin{array}{cc}
-\frac{c_{f}+c_{r}}{m v_{x}} & \frac{c_{r} l_{r}-c_{f} l_{f}}{m v_{x}}-v_{x} \\
\frac{c_{r} l_{r}-c_{f} l_{f}}{I_{z} v_{x}} & -\frac{c_{r} l_{r}^{2}+c_{f} l_{f}^{2}}{I_{z} v_{x}}
\end{array}\right], B=\left[\begin{array}{c}
\frac{c_{f}}{m} \\
\frac{c_{f} l_{f}}{I z}
\end{array}\right] E=\left[\begin{array}{c}
\frac{1}{m} \\
\frac{l_{w}}{I_{z}}
\end{array}\right]
$$

Note that, the tire model (2) is valid only in the case of low lateral acceleration. However, when lateral acceleration becomes high, the tire forces are no longer linear with respect to the wheel slip angles due to the tire saturation property. Accordingly, the cornering stiffness parameters $c_{f}$ and $c_{r}$ used in the linear tire model (2) will vary when the road friction changes or when the nonlinear tire domain is reached. Taking these variations into account, the following linear adaptive tire model is used to correct the cornering stiffness values via the uncertain variables $\Delta c_{f}$ and $\Delta c_{r}$ as

$$
F_{y f}=\left(c_{f 0}+\Delta c_{f}\right) \alpha_{f}, \quad F_{y r}=\left(c_{r 0}+\Delta c_{r}\right) \alpha_{r}
$$

where $c_{i 0}, \mathrm{i} \in\{r, f\}$, represents the known nominal value and $\Delta c_{i}, \mathrm{i} \in\{r, f\}$, is assumed to be unknown but bounded with a priori known bounds. Furthermore, it is clear that the uncertain bicycle model (4) is non-linear because of the term $\frac{1}{v_{x}}$. In this paper, the longitudinal velocity is considered piecewise constant and a switching rule depending on the measured velocity is deduced to select the active subsystem. Then, the uncertain non-linear bicycle model (4) is transformed into a switched uncertain discrete-time model ${ }^{1}$ :

$$
\left\{\begin{array}{l}
x_{k+1}=\left(A_{0, \sigma(k)}+\Delta A_{\sigma(k)}\left(\theta_{k}\right)\right) x_{k}+ \\
\left(B_{0, \sigma(k)}+\Delta B_{\sigma(k)}\left(\theta_{k}\right)\right) u_{k}+E_{\sigma(k)} d_{k} \\
y_{k}=C x_{k}
\end{array}\right.
$$

where $x_{k} \in \mathbb{R}^{n}, u_{k} \in \mathbb{R}^{m}, y_{k} \in \mathbb{R}^{p}, d_{k} \in \mathbb{R}^{q}$ are the state, the control input, the output vector and the unknown input respectively. $\sigma: \mathbb{R}^{+} \rightarrow \mathcal{I}=\{1,2, \ldots, N\}$ is the switching signal assumed to be available in real time. The matrices $A_{0, \sigma(k)} \in\left\{A_{0,1}, A_{0,2}, \ldots, A_{0, N}\right\}$ and $B_{0, \sigma(k)} \in\left\{B_{0,1}, B_{0,2}, \ldots, B_{0, N}\right\}$ are the state space matrices assumed to be constant and known a priori. $E_{\sigma(k)}$ and $C$ stand for the unknown input distribution matrix and the output equation matrix, respectively. The matrices $\Delta A_{\sigma(k)}\left(\theta_{k}\right) \in\left\{\Delta A_{1}\left(\theta_{k}\right), \Delta A_{2}\left(\theta_{k}\right), \ldots, \Delta A_{N}\left(\theta_{k}\right)\right\}$ and $\Delta B_{\sigma(k)} \in\left\{\Delta B_{1}\left(\theta_{k}\right), \Delta B_{2}\left(\theta_{k}\right), \ldots, \Delta B_{N}\left(\theta_{k}\right)\right\}$ are assumed to be unknown but bounded representing the uncertainty of the system caused by the time-varying parameter $\theta_{k}=$ $\left[\begin{array}{ll}\Delta c_{f} & \Delta c_{r}\end{array}\right]^{T} \in \Theta$, where $\Theta$ is an interval box given by:

$$
\Theta=\left\{\theta_{k} \in \mathbb{R}^{r} \mid \theta_{k}^{-} \leq \theta_{k} \leq \theta_{k}^{+}\right\}
$$

For easiness of further developments, the state space representation (6) is rewritten in the following equivalent form:

$$
\left\{\begin{array}{l}
x_{k+1}=A_{0, \sigma(k)} x_{k}+B_{0, \sigma(k)} u_{k}+E_{\sigma(k)} d_{k}+\delta_{\sigma(k)} \\
y(t)=C x(t)
\end{array}\right.
$$

where $\delta_{\sigma(k)}=\Delta A_{\sigma(k)}\left(\theta_{k}\right) x_{k}+\Delta B_{\sigma(k)}\left(\theta_{k}\right) u_{k}$.

\section{VEHICLE FAULT DETECTION}

\section{A. UI decoupling}

For the system (8), a switched observer that reconstructs the state $x_{k}$ without any information on the UI $d_{k}$ can be constructed using the input $u_{k}$ and the measured output $y_{k}$ as

$$
\left\{\begin{array}{l}
\hat{x}_{k+1}=N_{\sigma(k)} \hat{x}_{k}+K_{\sigma(k)} y_{k}+G_{\sigma(k)} u_{k}-H_{\sigma(k)} y_{k+1} \\
\hat{y}_{k}=C \hat{x}_{k}
\end{array}\right.
$$

with the initial state $\hat{x}_{0} \in \mathbb{R}^{n} . N_{\sigma(k)}, K_{\sigma(k)}, G_{\sigma(k)}$ and $H_{\sigma(k)}$ are gain matrices with appropriate dimensions to be determined.

Theorem 1. The switched observer (9) is a switched UIO

\footnotetext{
${ }^{1}$ The zero order hold method is used to obtain (6).
} 
for system (8) if and only if the following conditions are satisfied:

1) $e_{k+1}=N_{\sigma(k)} e_{k}+\Delta_{\sigma(k)}$ is ISS-stable under arbitrary switching;

2) $N_{\sigma(k)}=P_{\sigma(k)} A_{0, \sigma(k)}-K_{\sigma(k)} C$;

3) $P_{\sigma(k)} B_{0, \sigma(k)}-G_{\sigma(k)}=0$;

4) $P_{\sigma(k)} E_{\sigma(k)}=0$.

where $P_{\sigma(k)}=\mathcal{I}_{n}+H_{\sigma(k)} C$ and $\Delta_{\sigma(k)}=P_{\sigma(k)} \delta_{\sigma(k)}$.

Proof. The dynamics of the state estimation error $e_{k}=$ $x_{k}-\hat{x}_{k}$ is given by

$$
\begin{aligned}
e_{k+1} & =x_{k+1}-\hat{x}_{k+1} \\
& =\left(\mathcal{I}_{n}+H_{\sigma(k)} C\right) x_{k+1}-N_{\sigma(k)} \hat{x}_{k}-K_{\sigma(k)} y_{k}-G_{\sigma(k)} u_{k}
\end{aligned}
$$

By denoting $P_{\sigma(k)}=\mathcal{I}_{n}+H_{\sigma(k)} C$ and using (6), the dynamics of the state estimation error is

$$
\begin{aligned}
& e_{k+1}=\left(P_{\sigma(k)} A_{0, \sigma(k)}-K_{\sigma(k)} C\right) x_{k}-N_{\sigma(k)} \hat{x}_{k}+ \\
& \left(P_{\sigma(k)} B_{0, \sigma(k)}-G_{\sigma(k)}\right) u_{k}+P_{\sigma(k)} E_{\sigma(k)} d_{k}+P_{\sigma(k)} \delta_{\sigma(k)}
\end{aligned}
$$

If the following relationships hold

$$
\begin{gathered}
N_{\sigma(k)}=P_{\sigma(k)} A_{0, \sigma(k)}-K_{\sigma(k)} C \\
P_{\sigma(k)} B_{0, \sigma(k)}-G_{\sigma(k)}=0 \\
P_{\sigma(k)} E_{\sigma(k)}=0
\end{gathered}
$$

then, (10) is reduced to

$$
e_{k+1}=N_{\sigma(k)} e_{k}+\Delta_{\sigma(k)}
$$

which completes the proof.

From (12), one can see that $d_{k}$ has been decoupled under the conditions 2-4 of Theorem 1, but the effect of uncertainties $\Delta_{\sigma(k)}$ still persists. Therefore, the switched observer design consists on finding the observer gain $K_{\sigma(k)}$ such that the observer error dynamics (12) is asymptotically stable when $\Delta_{\sigma(k)}=0$ and is ISS-stable with respect to uncertainties when $\Delta_{\sigma(k)} \neq 0$.

\section{B. LMI formulation}

The Lemma 1 is useful for the proof of ISS-stability of the SUIO (9).

Lemma 1. The necessary and sufficient conditions for the existence of the SUIO (9) for the system (8) are:

1) $\operatorname{rank}\left(C E_{\sigma(k)}\right)=\operatorname{rank}\left(E_{\sigma(k)}\right)=q, \forall \sigma(k)$;

2) The pair $\left(P_{\sigma(k)} A_{0, \sigma(k)}, C\right)$ is detectable $\forall \sigma(k)$.

Remark 1. The first condition in Lemma 1 ensures that equation (11c) is solvable, and a particular solution can be given as follows:

$$
H_{\sigma(k)}=-E_{\sigma(k)}\left(\left[\left(C E_{\sigma(k)}\right)^{T}\left(C E_{\sigma(k)}\right)\right]^{-1}\left(C E_{\sigma(k)}\right)^{T}\right)
$$

Moreover, the second condition, sometimes referred as "strong detectability condition" is equivalent to that the transmission zeros from the UI to the output must be stable, i.e.

$$
\operatorname{rank}\left(\left[\begin{array}{cc}
s \mathcal{I}_{n}-A_{0, \sigma(t)} & E_{\sigma(k)} \\
C & 0
\end{array}\right]\right)=n+q,
$$

holds $\forall \sigma(k)$ and for all complex number $s$ with $\mathcal{R} e(s) \geq 0$. The next theorem provides sufficient conditions for the state estimation error (12) to be ISS-stable under arbitrary switching. Before proceeding, we begin by stating the following equivalence.

Lemma 2. The following conditions are equivalent:

$\left(E_{1}\right)$ There exists a symmetric matrix $Q$ such that

$$
\left[\begin{array}{cc}
N^{T} Q N+(\alpha-1) Q & N^{T} Q \\
Q N & Q-\gamma \mathcal{I}
\end{array}\right] \prec 0
$$

$\left(E_{2}\right)$ There exist a symmetric matrix $Q$ and a matrix $S$ such that

$$
\left[\begin{array}{ccc}
(\alpha-1) Q & 0 & N^{T} S^{T} \\
(*) & -\gamma \mathcal{I}_{n} & S^{T} \\
(*) & (*) & -S^{T}-S+Q
\end{array}\right] \prec 0
$$

Proof. If we apply the Schur complement with respect to the block $(3,3)$ of $(16)$, we recover directly (15) by choosing $S=S^{T}=Q$, hence $\left(E_{1}\right)$ implies $\left(E_{2}\right)$. Moreover, from the first block of (16), we have $(\alpha-1) Q \prec 0$. Then, multiplying (16) by $T=\left[\begin{array}{ccc}\mathcal{I}_{n} & 0 & N^{T} \\ 0 & \mathcal{I}_{n} & \mathcal{I}_{n}\end{array}\right]$ on the left and $T^{T}$ on the right, we get (15), which establishes that $\left(E_{2}\right)$ implies $\left(E_{1}\right)$ and the proof is complete.

Theorem 2. System (12) is uniformly ${ }^{2}$ ISS-stable with respect to the switching signal $\sigma(k)$ if there exist a symmetric matrix $Q$, matrices $S$ and $W_{i}, \forall i \in \mathcal{I}$, a constant $\gamma>0$ for given positive scalars $\alpha_{1}$ and $0<\alpha<1$ such that the following condition holds

$$
\begin{gathered}
\min _{Q, S, W_{i}} \gamma \\
{\left[\begin{array}{ccc}
(\alpha-1) Q & 0 & A_{0, i}{ }^{T} P^{T} S^{T}-C^{T} W_{i}^{T} \\
(*) & -\gamma \mathcal{I}_{n} & S^{T} \\
(*) & (*) & -S^{T}-S+Q
\end{array}\right] \prec 0}
\end{gathered}
$$

with $W_{i}=S K_{i}$ for any switching signal $\sigma(k)$. Furthermore the state estimation error (12) satisfies

$$
\lim _{k \rightarrow \infty}\left\|e_{k}\right\|_{2} \leq \sqrt{\frac{\gamma}{\alpha_{1}(1-\alpha)}}\left\|\Delta_{\sigma(k)}\right\|_{\infty}
$$

where $\Delta_{\sigma(k)}=P \delta_{\sigma(k)}$ and the maximum norm is given by $\left\|\Delta_{\sigma(k)}\right\|_{\infty}=\max \left\{\left|\Delta_{1}\right|,\left|\Delta_{2}\right|, \ldots,\left|\Delta_{N}\right|\right\}$.

Proof. For the stability analysis, we use the following common quadratic ISS-Lyapunov function

$$
V\left(e_{k}\right)=e_{k}^{T} Q e_{k}, \quad Q=Q^{T} \succ 0
$$

Setting $\Delta V\left(e_{k}\right) \triangleq V\left(e_{k+1}\right)-V\left(e_{k}\right)$, we have

$$
\Delta V\left(e_{k}\right)=e_{k+1}^{T} Q e_{k+1}-e_{k}^{T} Q e_{k}
$$

using (12), we get

$$
\begin{gathered}
\Delta V\left(e_{k}\right)=\left(N_{\sigma(k)} e_{k}+\Delta_{\sigma(k)}\right)^{T} Q\left(N_{\sigma(k)} e_{k}+\Delta_{\sigma(k)}\right)-e_{k}^{T} Q e_{k} \\
=e_{k}^{T} N_{\sigma(k)}^{T} Q N_{\sigma(k)} e_{k}+\Delta_{\sigma(k)}^{T} Q N_{\sigma(k)} e_{k} \\
\quad+e_{k}^{T} N_{\sigma(k)}^{T} Q \Delta_{\sigma(k)}+\Delta_{\sigma(k)}^{T} Q \Delta_{\sigma(k)}-e_{k}^{T} Q e_{k}
\end{gathered}
$$

${ }^{2}$ The word "uniformly" refers to uniformity with respect to switching signals. 
By adding and subtracting the terms $\alpha e_{k}^{T} Q e_{k}$ and $-\gamma \Delta_{\sigma(k)}^{T} \Delta_{\sigma(k)}$, we obtain that

$$
\begin{aligned}
\Delta V\left(e_{k}\right) & =\left[\begin{array}{c}
e_{k}^{T} \\
\Delta_{\sigma(k)}^{T}
\end{array}\right]\left[\begin{array}{cc}
N_{\sigma(k)}^{T} Q N_{\sigma(k)}+(\alpha-1) Q & N_{\sigma(k)}^{T} Q \\
(*) & Q-\gamma \mathcal{I}_{n}
\end{array}\right] \\
& \times\left[\begin{array}{c}
e_{k} \\
\Delta_{\sigma(k)}
\end{array}\right]-\alpha e_{k}^{T} Q e_{k}+\gamma \Delta_{\sigma(k)}^{T} \Delta_{\sigma(k)}
\end{aligned}
$$

Then, the satisfaction

$$
\left[\begin{array}{cc}
N_{\sigma(k)}^{T} Q N_{\sigma(k)}+(\alpha-1) Q & N_{\sigma(k)}^{T} Q \\
(*) & Q-\gamma \mathcal{I}_{n}
\end{array}\right] \prec 0
$$

is equivalent to satisfy (17) according to Lemma 2. Then, from (20) we get

$$
\Delta V\left(e_{k}\right)<-\alpha e_{k}^{T} Q e_{k}+\gamma \Delta_{\sigma(k)}^{T} \Delta_{\sigma(k)}
$$

that implies

$$
V\left(e_{k+1}\right)<(1-\alpha) V\left(e_{k}\right)+\gamma\left\|\Delta_{\sigma(k)}\right\|_{2}
$$

Integrating (23) over the interval $\left[k_{0}, k\right)$, we have

$$
V\left(e_{k}\right) \leq(1-\alpha)^{k-k_{0}} V\left(e_{0}\right)+\gamma \sum_{m=0}^{k-1}(1-\alpha)^{k-m-1}\left\|\Delta_{\sigma(m)}\right\|_{2}
$$

knowing that (19) satisfies for some $\alpha_{2}>\alpha_{1}>0$ the following inequality

$$
\alpha_{1}\left\|e_{k}\right\|_{2} \leq V\left(e_{k}\right) \leq \alpha_{2}\left\|e_{k}\right\|_{2}
$$

allows to deduce that

$$
\begin{aligned}
\left\|e_{k}\right\|_{2} \leq \frac{1}{\sqrt{\alpha_{1}}} & \left(\alpha_{2}(1-\alpha)^{k-k_{0}}\left\|e_{0}\right\|_{2}^{2}+\right. \\
& \left.\frac{\gamma}{1-\alpha} \sum_{m=0}^{k-1}[1-\alpha]^{k-m}\left\|\Delta_{\sigma(m)}\right\|_{2}^{2}\right)^{\frac{1}{2}}
\end{aligned}
$$

Hence, when $k \rightarrow \infty$ and using the maximum norm of $\Delta_{\sigma(k)},(18)$ is obtained which ends the proof.

Remark 2. The uniform ISS stability is usually used when there is no restriction on the switching signal $\sigma(k)$ and requires that all the subsystems are ISS-stable. This condition is ensured by the choice of the gain $K_{\sigma(k)}$. Note that the subsystems stability assumption is not sufficient to guarantee the stability of the overall switched system. However, the existence of the common quadratic ISS-Lyapunov function (19) is necessary and sufficient for the ISS-Stability of a the switched system (12) [13].

Remark 3. The existence of a common Lyapunov function for the switched system (12) is very conservative. The vehicle lateral dynamics system under consideration possesses a common Lyapunov function as will be shown in simulation, and remains stable under arbitrary switching signals. But despite this fact and in order to derive less conservative conditions our attention will be paid in future works on a less conservative class of Lyapunov functions, called multiple Lyapunov functions.

\section{Fault detection using interval model}

In the sequel, we are interested in actuator fault detection in vehicle lateral dynamics. To do this, the proposed switched UIO scheme is employed for actuator FD as described in the following. The fault detection procedure is based on testing whether the vehicle measured output $y_{k}$ is consistent with its estimation $\hat{y}_{k}$ provided by the UIO (9). When an inconsistency is detected, the existence of a fault is indicated. This consistency check is based on generating a fault indicator, known as residual $r_{k}$ and given by

$$
r_{k}=y_{k}-\hat{y}_{k}
$$

which can be also expressed using state estimation error $e_{k}$ as follows

$$
r_{k}=C e_{k}
$$

Note that the residual vector (28) is expected to be zero in fault-free case and deviate from zero in the presence of faults [1], [2]. However, due to the presence of parameter uncertainties $\Delta_{\sigma(k)}$, the residual vector $r_{k}$ will be different from zero, even in a non-faulty situation. We propose in this paper to use the so called passive fault detection method, that enhances the robustness of the fault detection schema at the decision making step. The initial state uncertainties and system parameter uncertainties are propagated to the residual vector, allowing to determine residual reachable interval. When the residual is outside the given interval, the occurrence of a fault is proved, i.e. while the residual

$$
r_{k} \in \star \mathbb{R}_{k}=\left[r_{k}^{-}, r_{k}^{+}\right]
$$

no fault occurred, and the residual value is simply due to the parameter uncertainty. $r^{-}$and $r^{+}$are lower and upper bounds of the residual $r_{k}$, respectively. The set $\star \mathbb{R}_{k}$ is obtained in real time using zonotopes.

\section{Implementation of the FD schema using zonotopes}

In this paper, zonotopes are used to implement the fault detection algorithm. The following definitions, assumptions and Lemma are introduced in advance.

Definition 1. The Minkowski sum of two sets $\mathbb{X}_{1}$ and $\mathbb{X}_{2}$ is given by $\mathbb{X}_{1} \oplus \mathbb{X}_{2}=\left\{x_{1}+x_{2}: x_{1} \in \mathbb{X}_{1}, x_{2} \in \mathbb{X}_{2}\right\}$.

Definition 2. [10] Given a center vector $x_{c} \in \mathbb{R}^{n}$ and a matrix $\mathcal{H} \in \mathbb{R}^{n \times m}$, the Minkowski sum of the segments defined by the columns of matrix $\mathcal{H}$ is called zonotope of order $m$. This set is defined by:

$$
\mathbb{X}=x_{c} \oplus \mathcal{H} \beta^{m}=\left\{x_{c}+\mathcal{H} z: z \in \beta^{m}\right\}
$$

where $\beta^{m}$ is an unitary box composed by $m$ unitary intervals. Definition 3. The interval hull $\star \mathbb{X}_{1}$ of a closed set $\mathbb{X}_{1}$ is the smallest interval box that contains $\mathbb{X}_{1}$.

Assumption 1. The initial state $x_{0}$ is inside an initial zonotope $\mathbb{X}_{0}$, implying that the initial state estimation error $e_{0}$ is inside the zonotope $\mathbb{E}_{0}=C \mathbb{X}_{0}$, i.e. $e_{0} \in \mathbb{E}_{0}$.

Assumption 2. It is assumed that the state and input vectors $x_{k}$ and $u_{k}$ are bounded as follows $x_{k}^{-} \leq x_{k} \leq x_{k}^{+}$, $u_{k}^{-} \leq u_{k} \leq u_{k}^{+}$, where, $x_{k}^{-}, x_{k}^{+}, u_{k}^{-}$and $u_{k}^{+}$are a priori known bounds reflecting the vehicle physical limits. 


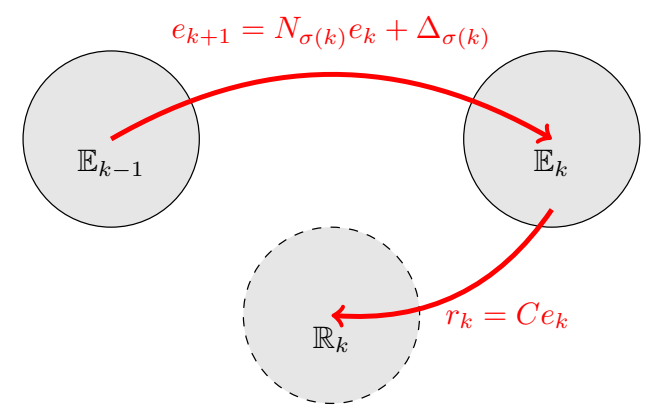

Fig. 1. Main steps for residual interval computation

Lemma 3. [4] Let the vector $x_{k} \in \mathbb{R}^{n}$ be a variable vector with given bounds $x_{k}^{+}, x_{k}^{-} \in \mathbb{R}^{n}$ such that $x_{k}^{-} \leq x_{k} \leq x_{k}^{+}$

1) If $M \in \mathbb{R}^{n \times n}$ is a constant matrix, then

$$
\bar{M} x_{k}^{-}-\underline{M} x_{k}^{+} \leq M x_{k} \leq \bar{M} x_{k}^{+}-\underline{M} x_{k}^{-}
$$

2) If $M \in \mathbb{R}^{n \times n}$ is a variable such that $M^{-} \leq M \leq M^{+}$ for some $M^{-}, M^{+} \in \mathbb{R}^{n \times n}$, then

$$
\begin{gathered}
\bar{M}^{-}{\overline{x_{k}}}^{-}-\bar{M}^{+} x_{x_{k}}^{-}-\underline{M}^{-}{\overline{x_{k}}}^{+}+\underline{M}^{+}{ }^{x_{k}}{ }^{+} \leq M x_{k} \leq \\
\bar{M}^{+} \bar{x}_{k}{ }^{+}-\bar{M}^{-} \underline{x k}^{+}-\underline{M}^{+} \bar{x}_{k}^{-}+\underline{M}^{-} \underline{x k}^{-}
\end{gathered}
$$

where $\bar{\star}=\max (0, \star)$ and $\star=\bar{\star}-\star$.

Note that since $\theta_{k} \in \Theta$, the system matrices $\Delta A_{\sigma(k)}\left(\theta_{k}\right)$ and $\Delta B_{\sigma(k)}\left(\theta_{k}\right)$ are bounded based on (7) as

$$
\begin{aligned}
& \Delta A_{\sigma(k)}^{-} \leq \Delta A_{\sigma(k)}\left(\theta_{k}\right) \leq \Delta A_{\sigma(k)}^{+} \\
& \Delta B_{\sigma(k)}^{-} \leq \Delta B_{\sigma(k)}\left(\theta_{k}\right) \leq \Delta B_{\sigma(k)}^{+}
\end{aligned}
$$

Therefore, using Assumption 2 together with (33) and applying Lemma 3 , the additive term $\Delta_{\sigma(k)}$ in (12) can be bounded by means of an interval box $\mathcal{D}$ given by:

$$
\begin{aligned}
\mathcal{D}=\left\{\Delta_{\sigma(k)} \in \mathbb{R}^{n} \mid \Delta_{\sigma(k)}^{-} \leq\right. & \Delta_{\sigma(k)} \leq \Delta_{\sigma(k)}^{+}, \\
& \left.\Delta_{\sigma(k)}^{-}, \Delta_{\sigma(k)}^{+} \in \mathbb{R}^{n}\right\}
\end{aligned}
$$

Furthermore, $\mathcal{D}$ can be rewritten as a zonotope $\mathcal{D}=\Delta_{c} \oplus$ $\mathcal{H}_{\Delta_{\sigma(k)}^{+}} \beta^{n}$, where $\mathcal{H}_{\Delta_{\sigma(k)}^{+}} \in \mathbb{R}^{n \times n}$ is a diagonal matrix with the main diagonal being $\Delta_{\sigma(k)}^{+}$and $\Delta_{c}$ is a known and constant vector.

Then, using Definitions 2 and 3, the state estimation error uncertainty is bounded using zonotopes and (12) is transformed to the following equivalent form

$$
\begin{gathered}
e_{k+1}^{c}=N_{\sigma(k)} e_{k}^{c} \\
\mathcal{H}_{k+1}=\left[\begin{array}{ll}
N_{\sigma(k)} \mathcal{H}_{k} & \mathcal{H}_{\Delta_{\sigma(k)}^{+}}
\end{array}\right]
\end{gathered}
$$

where $e_{k+1}^{c}$ and $\mathcal{H}_{k+1}$ are the center and segment matrix of $\mathbb{E}_{k+1}$, respectively.

The equivalent compact description of (35) is given as follows

$$
\mathbb{E}_{k+1}=N_{\sigma(k)} \mathbb{E}_{k} \oplus \mathcal{D}
$$

Thus, the residual zonotope at instant $k$ can be written as

$$
\mathbb{R}_{k}=C \mathbb{E}_{k}
$$

by using the iterative algorithm presented in Figure 1. The zonotope $\mathbb{R}_{k}$ is obtained for time instants $k>0$. Subsequently, the interval for residual vector $\star \mathbb{R}_{k}$ is obtained by computing the interval hull of the zonotope (37). By means of testing the robust FD criterion (29), the occurrence or absence of faults is detected in real time.

\section{Simulation RESUlTS USING REAL DATA}

In this section, the robust fault detection scheme proposed in Section III is applied to the actuator fault detection problem of vehicle lateral dynamic system described in Section II.

The real data used in the validation process are acquired using a prototype vehicle. The run was performed on a test track located in the city of Versailles-Satory (France). The track is $3.5 \mathrm{~km}$ length with various curve profiles allowing vehicle dynamics excitation. Several sensors are implemented on the vehicle. The yaw rate $r$ is measured using an inertial unit. An odometer is used to provide the vehicle longitudinal speed while the steering angle $\delta_{f}$ is measured by an absolute optical encoder. A wind lateral force acting as unknown input is added in simulation and presented in Figure 2. The steering angle, the yaw rate and longitudinal velocity profiles are plotted in Figure 3. Measured vehicle specifications for the simulation and experimental validation can be found in [6]. For the simulation scenario, three subsystems are defined for $v_{x}^{1}=8.5 \mathrm{~m} / \mathrm{s}, v_{x}^{2}=13.55 \mathrm{~m} / \mathrm{s}$ and $v_{x}^{3}=18.05 \mathrm{~m} / \mathrm{s}$. In addition, the following switching law is considered:

$$
\sigma(t)=\left\{\begin{array}{lll}
1 & \text { if } & v_{x} \in\left[V_{x}^{0}, V_{x}^{1}[\right. \\
2 & \text { if } & v_{x} \in\left[V_{x}^{1}, V_{x}^{2}[\right. \\
3 & \text { if } & v_{x} \in\left[V_{x}^{2}, V_{x}^{3}\right]
\end{array}\right.
$$

with $v_{x}^{k}=\frac{V_{x}^{k}-V_{x}^{k-1}}{2}$ for $k=1,2,3$. Furthermore, it is assumed that the cornering stiffness parameters are affected by $\pm 10 \%$ uncertainty in their nominal values.

The proposed algorithm is evaluated by introducing an actuator bias into the data provided by the encoder. Two fault zones are considered and represented with the switching signal in Figure 4. The first fault zone is in a stationary space where the second mode remains unchanged. The second zone is in a space where the vehicle dynamics switches quickly from the second to third mode and from the third to second mode, alternatively. According to the procedure described in Section III, the corresponding gain matrices of the UIO (9) for the lateral dynamics system (8) are designed. Due to space limitations, the gain matrices are omitted. The resulting attenuation level is $\gamma=0.0145$ for an actuator bias of $0.25 \mathrm{rad}, \alpha=0.5$ and a sampling time $T_{s}=0.6 \mathrm{~s}$.

Simulation results of the actuator fault detection are depicted in Figure 5. The gains which are obtained by solving the optimization problem in Theorem 2 allow to attenuate the effect of the uncertainties and the residual is almost zero. On the other hand, regarding the fault zone, where the vehicle dynamics switches alternatively between two modes, and the zone where no switch occurs, it can be noticed that the residual vector $r_{k}$ is outside the estimated set $\star \mathbb{R}$. The fault is well detected in both cases until it disappears. Furthermore, there is no delay in the fault indication. The fault detection accuracy is equal in both zones, and, the switching does not influence the detection quality. 


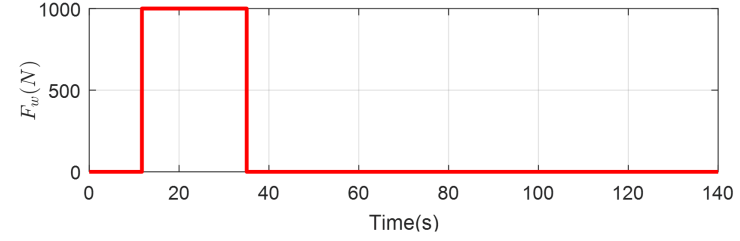

Fig. 2. Lateral wind gust input $F_{w}$.
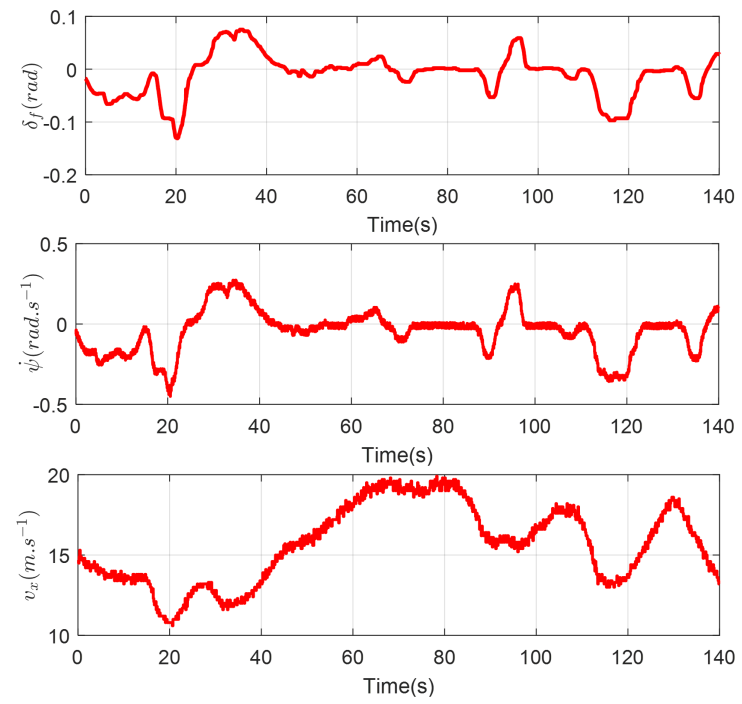

Fig. 3. Steering angle $\delta_{f}$, yaw rate $\dot{\psi}$ and longitudinal velocity $v_{x}$.

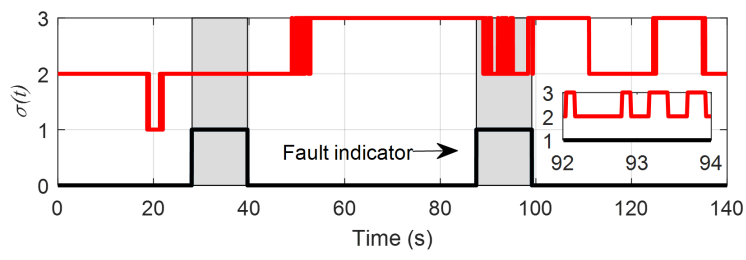

Fig. 4. Switching law and faults zone.

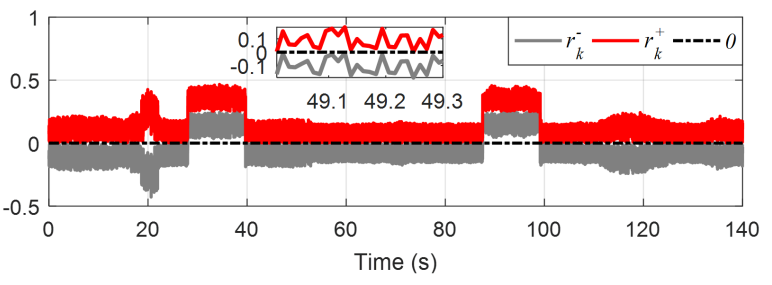

Fig. 5. Residue

\section{CONCLUSION}

In this paper, a new vehicle robust fault detection scheme, which combines switched UIO and zonotopes has been proposed. A multiple model switching structure is considered to take into account the longitudinal velocity variation. Then, Multiple SUIO-based FD are constructed to decouple the effect of UI from the residual vector. The gain matrices have been designed using a common ISS-Lyapunov function and an LMI formulation is obtained. As presented on simulation, the fault detection results are encouraging. The use of input-to-state stability concept guarantees uncertainties effect attenuation. Moreover, it has been shown that fast vehicle longitudinal velocity switching does not influence the fault detection quality.

Future work will be devoted to extend the fault detection method to fault isolation. Furthermore, another trivial extension of the method is to consider the variation of the longitudinal velocity and therefore adopt a Takagi-Sugeno representation of the vehicle lateral model. Reduction of the conservatism of the proposed LMIs using a multiple quadratic ISS-Lyapunov function will also be considered.

\section{REFERENCES}

[1] Abdelkader Akhenak, Mohammed Chadli, José Ragot, and Didier Maquin. Design of observers for takagi-sugeno fuzzy models for fault detection and isolation. IFAC Proceedings Volumes, 42(8):1109-1114, 2009.

[2] Jie Chen and Ron J Patton. Robust model-based fault diagnosis for dynamic systems, volume 3. Springer Science \& Business Media, 2012.

[3] Jie Chen, Ron J Patton, and Hong-Yue Zhang. Design of unknown input observers and robust fault detection filters. International Journal of control, 63(1):85-105, 1996.

[4] Denis Efimov, Leonid Fridman, Tarek Raissi, Ali Zolghadri, and Ramatou Seydou. Interval estimation for lpv systems applying high order sliding mode techniques. Automatica, 48(9):2365-2371, 2012.

[5] Pedro Guerra, Vicenc Puig, and Marcin Witczak. Robust fault detection with unknown-input interval observers using zonotopes. IFAC Proceedings Volumes, 41(2):5557-5562, 2008.

[6] Sara Ifqir, Naima Ait-Oufroukh, Dalil Ichalal, and Saïd Mammar. Robust interval observer for switched systems with unknown inputs : application to vehicle dynamics estimation. To be published in European Journal of Control [Preprint], 2018.

[7] Ari Ingimundarson, Jose Manuel Bravo, Vicenç Puig, Teodoro Alamo, and Pedro Guerra. Robust fault detection using zonotope-based setmembership consistency test. International journal of adaptive control and signal processing, 23(4):311-330, 2009.

[8] Rihab Lamouchi, Messaoud Amairi, Tarek Raissi, and Mohamed Aoun. Interval observer design for linear parameter-varying systems subject to component faults. In Control and Automation (MED), 2016 24th Mediterranean Conference on, pages 707-712. IEEE, 2016.

[9] Rihab Lamouchi, Messaoud Amairi, Tarek Raissi, and Mohamed Aoun. Actuator fault compensation in a set-membership framework for linear parameter-varying systems. IFAC-PapersOnLine, 50(1):40334038, 2017.

[10] Fatiha Nejjari, Vicenç Puig, Saúl Montes de Oca, and Atefeh Sadeghzadeh. Robust fault detection for lpv systems using interval observers and zonotopes. In Decision and Control, 2009 held jointly with the 2009 28th Chinese Control Conference. CDC/CCC 2009. Proceedings of the 48th IEEE Conference on, pages 1002-1007. IEEE, 2009.

[11] Tarek Raïssi, Gaétan Videau, and Ali Zolghadri. Interval observer design for consistency checks of nonlinear continuous-time systems. Automatica, 46(3):518-527, 2010.

[12] Rajesh Rajamani. Vehicle dynamics and control. Springer Science \& Business Media, 2011.

[13] RN Shorten and KS Narendra. On the stability and existence of common lyapunov functions for stable linear switching systems. In Decision and Control, 1998. Proceedings of the 37th IEEE Conference on, volume 4, pages 3723-3724. IEEE, 1998.

[14] Sébastien Varrier, Damien Koenig, and John J Martinez. Robust fault detection for uncertain unknown inputs lpv system. Control Engineering Practice, 22:125-134, 2014.

[15] Maiying Zhong, Steven X Ding, James Lam, and Haibo Wang. An lmi approach to design robust fault detection filter for uncertain lti systems. Automatica, 39(3):543-550, 2003. 